\title{
Patient Care Outcomes of the SEAHEC Improving Performance in Practice (IPIP) Experience
}

\author{
Albert A. Meyer, MD, Katrina E. Donabue, MD, MPH, Sonali Batish, MPH, \\ Terry Gentry, BS, Ashley Adams, BA, Amanda Brown, BS, and Melissa Baumann, BS
}

Purpose: This study describes the effects of the collaboration between the South East Area Health Education Center and Improving Performance in Practice (IPIP) on the improvement in quality markers in chronic disease states in a southeastern North Carolina family practice.

Methods: Teams were created throughout 6 counties to implement strategies at biweekly quality team meetings that would ultimately improve patient quality, as measured by adherence to IPIP benchmarks. Grant-funded cash incentives were given to the practice to create a chronic care registry. Quarterly learning network meetings encouraged the exchange of quality improvement techniques.

Results: The practice's quality team succeeded in creating a chronic care registry, improving patient care as measured by benchmark chronic disease states, and extending this knowledge to other areas of their practice.

Discussion: With mounting economic barriers to quality preventive services and medical access of any kind, the quality improvement in diabetic care and asthma is notable. The SEAHEC collaborative helped the practice achieve better results in approaching quality benchmarks than state averages, despite serving a more economically challenged population. (J Am Board Fam Med 2013;26:16-23.)

Keywords: Chronic Disease, Patient Care, Quality Improvement, Registries

Chronic disease management is challenging for primary care providers. The Centers for Disease Control and Prevention reports that, as recently as 2005, almost 1 in 2 American adults suffer from a chronic illness. ${ }^{1}$ These patients present a special burden on the health care system, accounting for $83 \%$ of our national health care spending. ${ }^{2}$ Especially troubling is the difficultly in identifying best practices for chronic disease management. A sys-

This article was externally peer reviewed.

Submitted 1 March 2012; revised 10 September 2012; accepted 17 September 2012.

From the South East Area Health Education Center, Wilmington, NC (AAM, SB, AA); Coastal Family Medicine, Wilmington, NC (AAM, TG); New Hanover Regional Medical Center, Wilmington, NC (AAM); the Department of Family Medicine, University of North Carolina at Chapel Hill, Chapel Hill (KED); and the Department of Clinical Research, University of North Carolina at Wilmington, Wilmington (AB, MB).

Funding: none.

Conflict of interest: none declared.

Corresponding author: Albert A. Meyer, MD, Family Medicine Residency Program, New Hanover Regional Medical Center, South East Area Health Education Center, 2523 Delaney Avenue, Wilmington, NC 28403 (E-mail: Albert.Meyer@seahec.net). tematic review of informational and behavioral interventions to improve chronic care suggested that although several different practice models were effective in improving medication adherence, clinical outcomes rarely were significantly improved. ${ }^{3}$ Even when outcomes may be improved, Medicare expenditures often are not reduced. ${ }^{4}$

Considering that a strong secondary goal to improved patient outcomes is reduced cost, it is concerning that even comprehensive interventions have minimal effects. ${ }^{5}$ This may be partially attributed to the systematic failure to publish improvement experiences, both positive and negative. ${ }^{6}$

\section{Improving Performance in Practice}

One initiative aimed at improving chronic care and patient outcomes is Improving Performance in Practice (IPIP). Details of the North Carolina (NC) IPIP program have been published previously, but we review them here briefly. ${ }^{5}$ The goal of the NC IPIP is to improve care in primary care practices by defining quality measures combined with the use of registries and development of learning 
networks. The NC IPIP relies on the collaboration of the statewide NC Area Health Education Center (AHEC) and Community Care of NC. Both organizations have regional offices across the state and are committed to supporting primary care practices and improving quality of care. These organizations offer practices help with data systems, provide a quality improvement consultant (QIC) to facilitate new initiatives within the practice, and offer continuing medical education and maintenance of certification part IV to participating physicians. ${ }^{7}$

The QIC is a local consultant who works closely with a practice, familiarizing themselves with the office protocols and work flows and developing data reporting systems. They guide the practice in setting priorities and goals for improved patient care. In addition, the QIC encourages practices to form a quality team, which usually is headed by a practice champion and members of the practice including both clinical and administrative staff. The QIC meets with the practice regularly and devises strategies to build patient registries for the chronic illness being followed and to devise methods of reporting data. During monthly meetings the QIC discusses progress, barriers to improvement, and goals for the practice based on identified practice resources to improve quality. The IPIP QIC also works with the local Community Care of NC regional office to set up quarterly collaborative meetings, which are learning networks for the participating practices. The QIC encourages representatives of each practice to attend the collaborative meetings. ${ }^{7}$

This article describes the experiences of Coastal Family Medicine (CFM), a family medicine teaching practice with 14 residents, 5 faculty physicians, and 1 faculty pharmacist with a primarily outpatient population, in their attempts to reach the goals set by IPIP and maintain improved chronic care. CFM works in conjunction with a QIC from the South East AHEC (SEAHEC), which is partnered with the Community Care of the Lower Cape Fear. ${ }^{7}$

\section{Methods}

It was decided by the regional IPIP initiative that enrolled practices, including CFM, would concentrate on diabetic measures that, when improved, lower emergency department visits and inpatient hospitalizations in addition to improving the qual- ity of life for those affected by the condition. ${ }^{8}$ Interventions implemented upon joining the IPIP initiative were monthly quality improvement meetings within the practice, participation in quarterly collaborative meetings, and the initiation of a chronic disease registry for diabetic patients. These measures were evaluated between July 2010 and June 2011. In addition, each intervention was examined for its sustainability.

\section{Collaborative Meetings}

The IPIP initiative in NC also includes the organization of quarterly meetings for all practices participating in each regional IPIP collaborative. CFM participates in quarterly meetings organized and hosted by SEAHEC, the regional AHEC, and the local Community Care of NC Medicaid network. Representatives from each practice are encouraged to attend the collaborative meetings. Practice representatives share barriers to quality enhancements and successful quality initiatives and provide novel approaches that can be used by other practices dealing with similar barriers to quality. Regional and national IPIP leaders are invited to the quarterly meetings to present new ideas or approaches to practice quality and to inject lessons learned in their own pursuits of quality. In addition, as an incentive to participation in the IPIP program, clinical participants may qualify for maintenance of certification part IV.

Although theoretically advantageous, these meetings can help the practice identify new procedures and improve current procedures only if the practice's physicians attend. It was determined that the direct impact of the collaborative meetings could be measured both by CFM physician attendance and the attempted implementation of any best practice reported by other regional practices.

\section{Biweekly Quality Team Meetings}

To directly involve all members of the staff in these quality improvement initiatives, a quality team was formed and a time for a biweekly meeting was established. At quality team meetings, representatives from the clerical staff, medical assistants, nurses, residents, faculty, the IPIP QIC, and the practice manager discuss quality improvement goals and Plan, Do, Study, Act (PDSA) cycles are suggested. These ongoing biweekly meetings provide a forum in which plans can be tried, evaluated, implemented, or discarded. Practice meetings are 
scheduled regularly on the second and last Thursday of each month. The effectiveness of monthly meetings was measured in the implementation of both successful and unsuccessful PDSA cycles.

\section{Registry}

Like many practices in the collaborative, an initial barrier faced by CFM was the inability to develop an effective registry for any disease or preventive service category. Despite having an electronic health record (EHR) for more than a decade and numerous attempts to form a consistent registry, data could not be accessed because faculty and residents would store benchmark data in different parts of the EHR. When it became obvious that the EHR had little capability to form a registry, external data storage ports were identified. In 2008, the Governor's Quality Initiative provided support to funnel data to a central registry. Because CFM is a hospitalbased clinic, there were months of negotiations and meetings to create a secure data transfer system on which the involved parties could agree, despite cash incentives given by IPIP in 3 milestone-based payments in the amounts of $\$ 1000, \$ 1000$, and $\$ 500$ to offset the time that practices required to set up registries. Data transfer and registry function began in February 2009 and continued through November 2009. The monthly data report allowed CFM to spot trends in the practice and set up creative PDSA cycles to improve quality. It seemed that positive results were inevitable; however, grant support for the central registry initiative vanished and a new data repository was needed. With the support of the IPIP quality coaches, the Reach My Doctor (RMD) online data storage site (http:// nc.rmdnetworks.com) was identified and hospital negotiations to accept this as a secure site were less formidable.

Patients initially were identified using specific diagnosis codes and visit parameters. The flow sheet served as a visit planner, tracking aspects of care that were overdue and dates when particular aspects of care were last completed. The provider marked care that was provided on the flow sheet, and these data were entered back into the RMD repository so that an updated flow sheet could be generated at the patient's next appointment. At the end of each month, RMD produced a summary report of each diabetic and asthma measure on a clinic-wide basis. These data were translated into a graphical form and presented to the clinic to iden-
Table 1. Improving Performance in Practice (IPIP) Goals and Clinic Measures

\begin{tabular}{|c|c|c|c|}
\hline & $\begin{array}{l}\text { IPIP } \\
\text { Goal }\end{array}$ & $\begin{array}{l}\text { July } \\
2010\end{array}$ & $\begin{array}{l}\text { June } \\
2011\end{array}$ \\
\hline \multicolumn{4}{|l|}{ Alc } \\
\hline Alc documented & $>90$ & 86 & $95^{*}$ \\
\hline$<7.0$ & $>75$ & 48 & 42 \\
\hline$>9.0$ & $<5$ & 29 & 26 \\
\hline \multicolumn{4}{|l|}{ Cholesterol } \\
\hline LDL documented & $>90$ & 78 & 82 \\
\hline$<100$ & $>50$ & 47 & 42 \\
\hline$<130$ & $>90$ & 65 & 67 \\
\hline \multicolumn{4}{|l|}{ Specialty care } \\
\hline Aspirin usage (age $\geq 40$ years) & $>85$ & $92^{*}$ & $95^{*}$ \\
\hline Influenza vaccination & $>75$ & $76^{*}$ & $88^{*}$ \\
\hline Foot exam & $>90$ & 88 & $93 *$ \\
\hline Kidney assessment & $>90$ & 90 & $94^{*}$ \\
\hline Set self-management goals & $>90$ & 89 & $94^{*}$ \\
\hline \multicolumn{4}{|l|}{ Blood pressure } \\
\hline Blood pressure documented & $>80$ & $93 *$ & $98^{*}$ \\
\hline$\leq 130 / 80$ & $>70$ & 45 & 36 \\
\hline$\leq 140 / 90$ & $>75$ & 68 & 62 \\
\hline \multicolumn{4}{|l|}{ Retinal } \\
\hline Eye exam & $>80$ & 67 & 68 \\
\hline \multicolumn{4}{|l|}{ Tobacco use } \\
\hline Assess smoking status & $>80$ & $90^{*}$ & $97^{*}$ \\
\hline Counseled about smoke/exposure & $>90$ & $100^{*}$ & $100^{*}$ \\
\hline
\end{tabular}

Values provided as percentages.

${ }^{*}$ Clinic measure above IPIP goal.

tify areas for potential PDSA cycles. In addition, for high-risk diabetic patients, lists stratified by patient risk were produced on a regular basis and made tracking care easier. Patients who were delinquent on preventive care were contacted and asked to come to the clinic for care.

The chronic disease quality measures used to assess the impact of all quality improvement interventions for diabetes along with IPIP goals are outlined in Table 1. The ultimate efficacy of any and all interventions, PDSA cycles, and monthly and quarterly meetings can be assessed with the final outcomes measured by the registry.

\section{Results}

\section{Collaborative Meetings}

The quarterly collaborative meetings have been successful as measured in terms of attendance. CFM faculty and administration have attended all but one of the quarterly collaborative meetings in the last 2 years and have provided positive feed- 
back. In addition, the practice has been asked to share their successes and feedback at several of the meetings. After each meeting, feedback is gathered via an online survey tool to measure the quality of the meeting and topics of interest for future meetings. These responses help to identify areas of mutual interest and determine future guest speakers and partner organizations that practices view as resources for improvement. Speakers from the University of North Carolina have spoken to the collaborative about topics such as open access scheduling, the use of registries to improve care, achieving designation as a patient-centered medical home, using the EHR as a tool for quality improvement, and goal setting for the diabetes and asthma group. Speakers from designated practices in the collaborative also present on topics such as, "How IPIP was Improving Our Practice," "Smoking Cessation Strategies," "Group Medical Visits For Diabetic Patients," and "How Our Practice Has Improved Foot Examination Rates." Other HER-related topics presented include "E-prescribing," "Health Information Exchange," and "Economic Stimulus Money and Meaningful Use," among others.

Although not as prolific in generating specific ideas for practice interventions, best practices were shared among regional practices as well. For example, one pediatric practice presented how they used their registry to track, contact, and improve their rate of influenza vaccination among patients with asthma. As a result, CFM queried patients with asthma from its RMD registry and reached out to them about receiving the influenza vaccine.

Because the IPIP initiative is not run by CFM, but by organizations at the state level, the burden of sustainability is not on the practice itself. SEAHEC does report that the recent addition of local sponsors covers the entire cost of the meetings and the meetings no longer rely on the IPIP grant to sustain their cost. In addition, the practice has been represented by a faculty physician at $100 \%$ of the collaborative meetings during the time frame of the study. This information suggests both that the meetings themselves will be sustained and that faculty physicians will continue to participate in these meetings.

\section{Biweekly Quality Team Meetings}

There have many successful PDSA cycles as a direct consequence of the weekly quality team meetings. From July 1, 2010, to June 30, 2011, 11 PDSA cycles were implemented. Four of these cycles are detailed below.

On numerous occasions, the nurse would frequently be contacted by frustrated patients with limited access to quality care. Issues with job satisfaction made it difficult to sustain a consistent person in the position. At quality team meetings, it was decided to eliminate the triage phone and divide the practice into 4 clinical teams, with the medical assistant service as the pacesetter for each team. The medical assistant would be assigned 4 or 5 doctors and would process phone messages, handle refills, and get to know the patients on that team. To define teams, it was necessary to establish formal patient panels for each team. Since the practice opened in 1997, more than 22,000 patients were in the computer even though most had not been seen in $>3$ years. These patients were given an inactive status and not assigned to a team. Faculty and residents were given lists of their active patients and were given the opportunity to review the lists and eliminate a patient who had moved, died, or transferred care. This improved access because active patients were given visit priority and panels clarified clinical population goals for the practice. It also greatly affected "no show" rates, plunging from a high of $21 \%$ to a current $16 \%$.

With better-defined panels, individual providers could use the clinic's registry to look at their diabetic patients and see which patients were not meeting IPIP benchmarks. A PDSA cycle was designed to identify all patients with a hemoglobin A1C $>9 \mathrm{mmol} / \mathrm{mol}$. Team nurses contacted these patients and scheduled an appointment with their doctor, PharmD provider or a designated nurse. For patients with access and adherence challenges the team proposed a designated nurse visit to review medication adherence, draw laboratory specimens, do foot examinations, develop patient selfmanagement goals, and arrange eye examinations. Standing orders were developed for the 3 registered nurses performing this patient service to generate data that would help the provider make clinical therapeutic decisions at the next visit. At the beginning of the time frame of the study, CFM was only at goal on 5 measures, which improved to being at goal on 9 measures (Table 1). While reaching goals for preventative screening is improving, CFM still is facing a considerable challenge in reducing the percentage of patients with a hemoglobin A1C $>9 \mathrm{mmol} / \mathrm{mol}$ (Figure 1). 
Figure 1. Patients with hemoglobin A1c $>9 \mathrm{mmol} / \mathrm{mol}$. SEAHEC, South East Area Health Education Center; IPIP, Improving Performance in Practice.

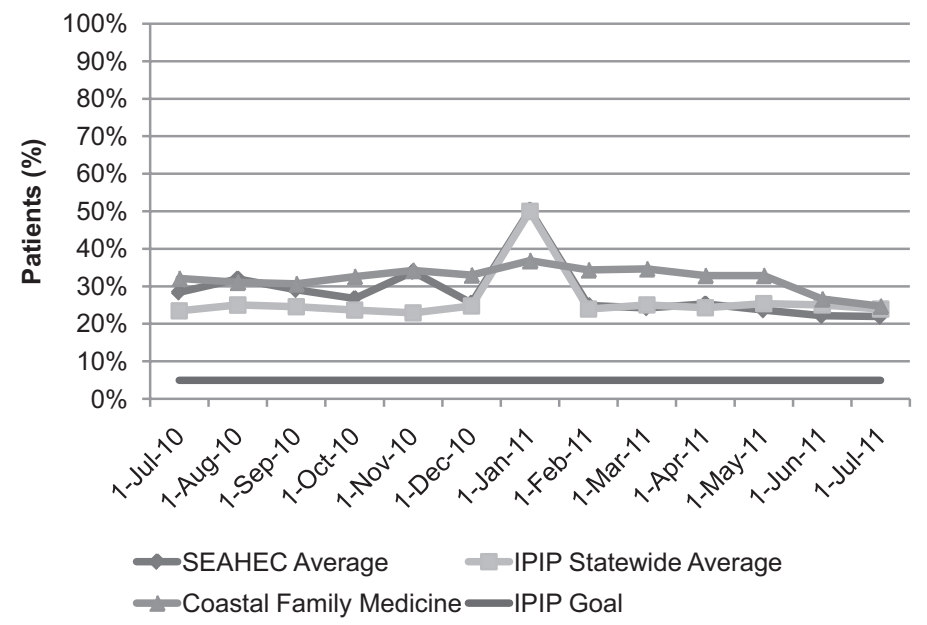

Another PDSA cycle sought to increase the documentation of hemoglobin A1C among the clinic's diabetic patients. A point-of-care (POC) hemoglobin A1C machine, financed by the aforementioned money from the IPIP incentive, was purchased and is available for POC testing at most visits. With this POC machine, the percentage of patients with a current documented hemoglobin $\mathrm{A} 1 \mathrm{C}$ is $98.5 \%$, up from $86.04 \%$ in July 2010 (Figure 2). As a result of this success, a POC low-density lipoprotein machine is being evaluated for purchase.

At one quality team meeting, it became apparent that no system existed to track who received an ophthalmologic annual referral. Referral tracking was taken on as a quality initiative, and college interns, with the guidance of the practice manager, devised a database system that coordinated the referrals. The access database tracked the referral from the point at which it was ordered until the consult was received. Patients that did not show for the appointment or did not go to their specialist appointments were identified and counseled. In addition, before the patient's follow-up appointment at CFM, consult reports were tracked, scanned, and made available for the provider to discuss with the patient. This has helped with the documentation for ret-

Figure 2. Patients with documented hemoglobin A1c. SEAHEC, South East Area Health Education Center; IPIP, Improving Performance in Practice.

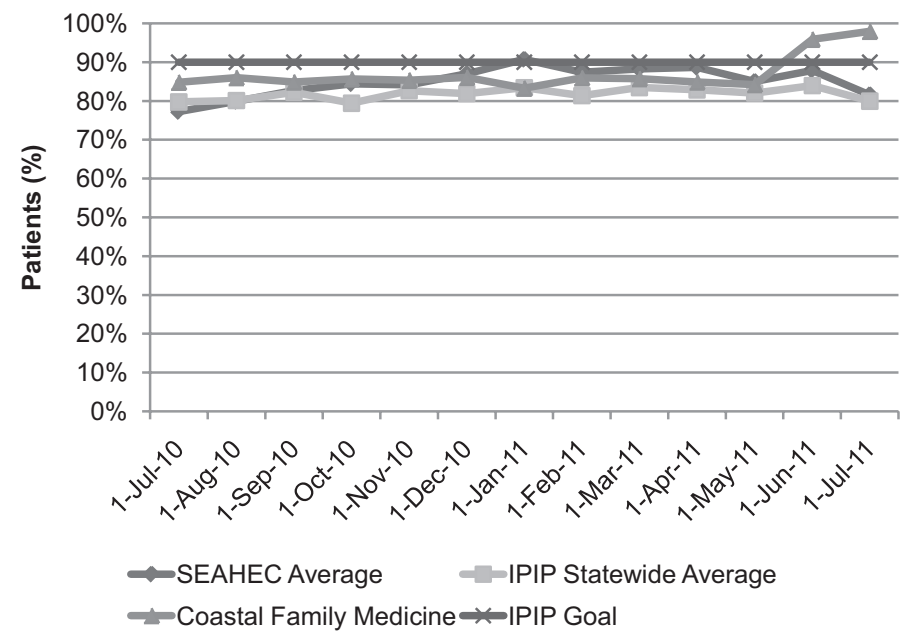


Figure 3. Patients with documented retinal screening. SEAHEC, South East Area Health Education Center; IPIP, Improving Performance in Practice.

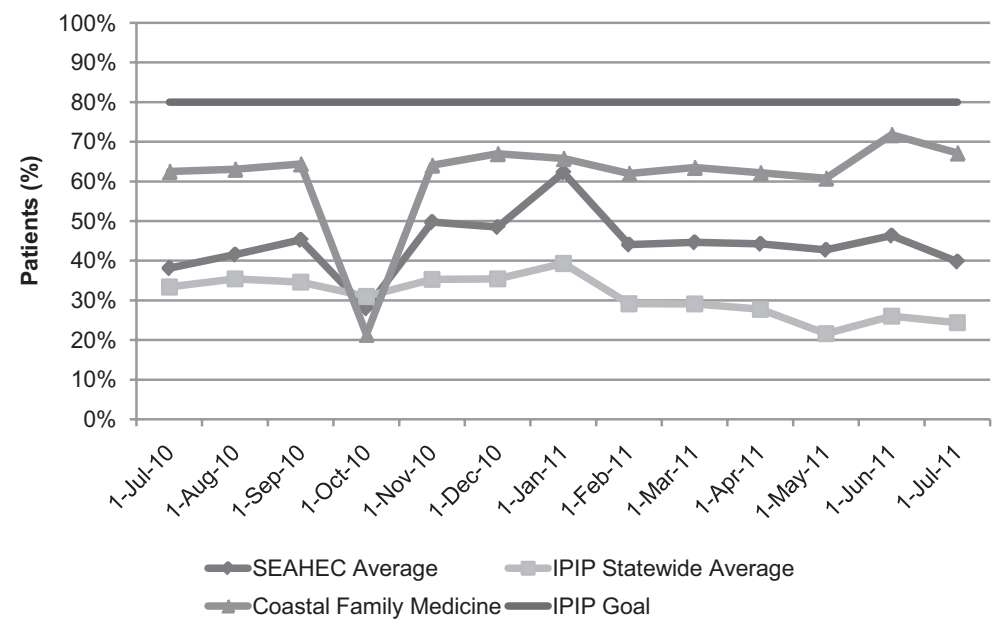

inal screening for diabetic patients and has allowed CFM to rely on other specialists to help integrate care. The percentage of patients with diabetes mellitus receiving eye examinations was $43 \%$ higher than the statewide average in July 2011 (Figure 3).

A secondary concern, but of equal importance, is the sustainability of this practice. During the time frame between June 2010 and July 2011, biweekly quality team meetings were held at regular intervals and were rarely missed. In addition, during this time, there were changes in faculty and resident physicians and administrative changes in the local QIC administration. The ability to overcome these barriers suggests there is no reason to believe that sustainability is not achievable.

\section{Registry}

Although PDSA cycles and best practices are excellent tools in reaching the chronic care benchmarks set by IPIP, the truest measure of the effectiveness of these interventions is to actually review patient outcomes and how they compare with current goals. There are measures that are still not at goal, but there has been improvement in 11 of the 17 diabetic goals set by IPIP during the study time frame (Table 1). The most marked improvement has been the $11 \%$ increase in those receiving the influenza vaccine and the $8 \%$ improvement in those who had a documented hemoglobin A1C level.

Thus far, the registry has been sustainable, first through the assistance of staff, then, when the staff was overburdened, the local university provided suitable unpaid interns seeking health administration experience to enter data into RMD. In the coming months, the practice will go live with a new, systemwide EHR, which will negate the need for the registry in its current form. The EHR then will be sustainable by virtue of its system-wide usage, and further updates and potential obstacles will be dealt with by the local health care system. Custom report templates allow for the extraction of IPIP data directly from the HER, and monthly benchmarking and trending reports will continue to be made available to practices during the quality meetings.

\section{Discussion}

Although some studies have shown that registries have not reduced costs of chronic care,${ }^{10}$ systematic reporting of data can be helpful to practices to see where improvement is needed. At CFM, this registry has allowed the practice to determine which areas need PDSA cycles and to review systematically the success or failure of its interventions.

This information has been vital enough that CFM has expanded it to other patient populations. In addition to these diabetic initiatives, CFM faculty and senior residents started an asthma registry in 2010. CFM also is focusing on its Well Child Check Benchmark. Reminder letters are being sent to the guardians of children in need of a well-child check, requesting they schedule an appointment. The practice stands at $83 \%$ compliance with 772 children enrolled in the registry, which exceeds the 
Centers for Medicare \& Medicaid Services benchmark of $80 \%$.

However, this experience may be limited for other practices because CFM is a family medicine residency program and, as such, has different characteristics from other family practices. A recent study in which program directors were interviewed about their perspectives on chronic care when training residents suggests that more challenges are present in residency programs than in traditional practices. The program directors cited the continuous rotation of physicians, limited time of faculty to research and implement new best practices, and resistance from residents as barriers to improvements in chronic disease care. ${ }^{11}$ Despite these difficulties, residents reported easier access to detailed information about chronic illness, increased selfmanagement support (Figure 4), and increased ability to consult current guidelines during a patient visit when Chronic Care Model elements were implemented for improving care for patients with asthma. ${ }^{12}$

CFM also has the support of local organizations that promote the education of physicians and improvement of patient care outcomes. SEAHEC is in a unique role in that it employs the local QIC, organizes the quarterly collaborative meetings, and oversees the residency education of the residents at CFM.

There are many other factors that allow CFM to pursue and sustain its quality improvement ambitions. The practice manager fosters collaboration among clinical staff, who are paid by the University of North Carolina, Chapel Hill, and the support staff, who are paid by the affiliated hospital. CFM is

\section{Figure 4. Patients with a documented self-management goal. SEAHEC, South East Area Health Education Center; IPIP, Improving Performance in Practice.}

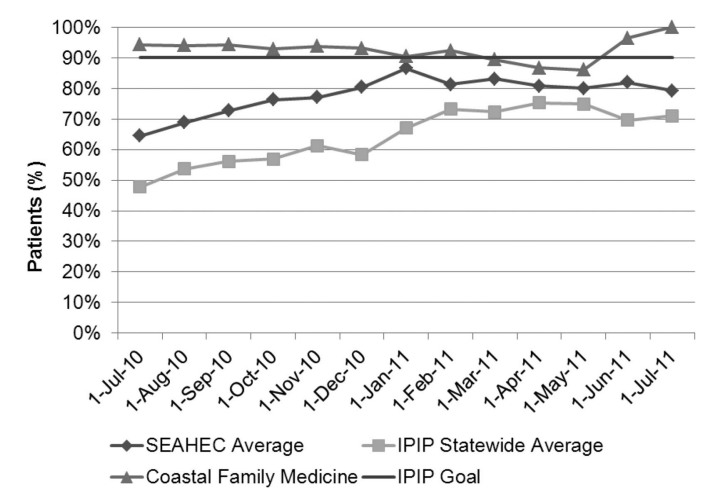

fortunate to have a doctor of pharmacy faculty member who has championed group diabetic visits and has taken on the most difficult patient care management issues with individual one-on-one pharmacy education visits. In addition, the practice has the use of well-trained, experienced registered nurses and medical assistants who provide nurse visits to patients that need extra education and counseling.

With the use of registries and clinical teams that ensure optimal care and coordination surrounding each patient, the practice decided to pursue $\mathrm{Na}$ tional Committee for Quality Assurance designation as a patient-centered medical home. Despite an EHR that could not pull quality reports, the practice highlighted its effective use of patient registries and tracking databases, as well as its welldocumented protocols for care that have led to practice-wide quality improvement. The process of assembling the application was approached as a PDSA cycle in which room for change and evolution in the process is considered. As with many of the aforementioned initiatives, a team was assembled to work toward patient-centered medical home designation. Each practice team took on a particular standard of the application. In addition, a core team with a physician champion, practice administrator, billing manager, and IPIP QIC tracked and coordinated the process. In April 2012, the practice was awarded designation as a level $2 \mathrm{pa}-$ tient-centered medical home, according to the 2011 standards.

\section{References}

1. Wu SY, Green A. Projection of chronic illness prevalence and cost inflation. Santa Monica, CA: RAND Health; 2000. Available at: www.cdc.gov/chronic disease/overview/index.htm. Accessed May 20, 2012.

2. Anderson G. Chronic conditions: making the case for ongoing care. Baltimore, MD: John Hopkins University; 2004. Available at http://www.partnership forsolutions.org/DMS/files/chronicbook2004.pdf. Accessed November 16, 2012.

3. Kripalani S, Xiaomei Y, Haynes B. Interventions to enhance medication adherence in chronic medical conditions. Arch Intern Med 2007;167:540-50.

4. Peikes D, Chen A, Schore J, Brown R. Effects of care coordination on hospitalization. quality of care, and health care expenditures among medicare beneficiaries. JAMA 2009;301:603-18.

5. Bernstein J. The elusive benefits of chronic care management. Arch Intern Med 2011;171:466-7. 
6. Davidoff F, Batalden P. Toward stronger evidence on quality improvement. Draft publication guidelines: the beginning of a consensus project. Qual Saf Health Care 2005;14:319-25.

7. Newton W, LeFebvre A, Donahue K, Bacon T, Dobson A. Infrastructure for large-scale quality-improvement projects: early lessons from North Carolina Improving Performance in Practice. J Contin Educ Health Prof 2010;30:106-13.

8. Improving Performance in Practice. Measures and standards. Available at: ipipprogram.org/measures. html. Accessed June 5, 2012.

9. Meyer A, Gentry T. The "no" show to the "yes" showhow our team transformed our practice. Presented at the
Society of Teachers of Family Medicine Conference on Practice Improvement: Constructing the Medical Home, November 5-8, 2009, Kansas City, MO.

10. Fireman B, Bartlett J, Selby J. Can disease management reduce health care costs by improving quality? Health Aff (Millwood) 2004;23:63-75.

11. Feifer C, Mora A, White B, Barnett BP. Challenges to improving chronic care disease care and training in residencies. Acad Med 2006;81:696-701.

12. Greene J, Rogers V, Yedidia M. The impact of implementing a chronic care residency training initiative on asthma outcomes. Acad Med 2007;82: $161-7$. 